\title{
A xenobiotic-free culture system for human limbal epithelial stem cells
}

\section{Maria Notaralt, David B Haddow? Shila MadNe| ${ }^{3}$ \& JulieT Danids'}

${ }^{\dagger}$ Author for correspondence IU CL Institute of O phthalmology, Cells for Sight Transplantation and Research Programme, 0 cular Repair and Regeneration Biology U nit, 11-43 Bath Street, London, EC1V 9EL, UK

Tel.: +44 207608 6969;

Fax: +44 207608 6887;

E-mail: m.notara@ud.ac.uk;

j.daniels@ucl.ac.uk

${ }^{2}$ CellTran Ltd, Innovation

Centre, 217 Portobello,

Sheffield, S1 4D P, UK

E-mail: d.b.haddow@ sheffield.ac.uk

${ }^{3} \mathrm{~K}$ roto Institute, Centre for

Biomaterials and Tissue engineering, $D$ epartment of Engineering $M$ aterials, $N$ orth Campus, Sheffield, S3 7H Q, UK

E-mail: S.M acneil@ sheffield.ac.uk

\footnotetext{
Keywords cell transplantation, feeder layer, human fibroblasts, limbal epithelium, stem cells, xenobiotic system

\begin{abstract}
Aims: Murine 3T3 feeder cells are commonly used for stem cell expansion. Although 'feeder-free' systems are being developed for a variety of stem cells including embryonic, the use of feeder cells currently remains optimal for the expansion of epithelial stem cells. In this study, M RC-5, a human embryonic fibroblast cell line, has been investigated for its potential use as a feeder layer in human limbal epithelial (HLE) cell expansion under serum-free conditions, with the aim of developing a xenobiotic-free culture system for therapeutic corneal regeneration applications. Materials and Methods: MRC- 5 feeder cells were compared with J2 $3 \mathrm{~T} 3$ mouse fibroblasts, in both serum-supplemented and serum-free conditions, in terms of their relative ability to support HLE cell metabolic activity, expression of the putative stem cell markers ABCG2 and P63 $\alpha$, cell differentiation using the cornea-specific cytokeratin 3 antibody and colony-forming efficiency.

Results: The proportion of HLE stem cells maintained was determined by functional colony-forming efficiency assays. The metabolic activity results showed that HLE cells cultured on MRC-5 fibroblasts under serum-free conditions proliferated as well as cells cultured on $\mathrm{J} 2$ cells under serum-free conditions. Moreover, the HLE cultured on MRC-5 fibroblasts under serum-free conditions expressed high levels of putative stem cell markers ABCG2 and P63 $\alpha$ and low levels of the differentiation marker CK3, indicating that they retained poorly differentiated 'stem cell-like' characteristics under those culture conditions. Clonal analysis of HLE cells cultured on growth-arrested feeder layers of $\mathrm{J} 2$ and MRC-5 fibroblasts showed that cells expanded on MRC- 5 and $\mathrm{J} 2$ fibroblasts in serum-free conditions had a colony-forming efficiency of approximately $1.5 \%$, indicating the maintenance of stem cells. Conclusions: These results demonstrate feasibility of expanding HLE cells for clinical purposes by using a human fibroblast cell line as a feeder layer, avoiding the use of bovine serum, while preserving the proliferative potential and stem cell characteristics of HLE cells.
\end{abstract}

The field of regenerative medicine offers the possibility of delivering new stem cell-based therapies for the treatment of diseases including Type II diabetes, Parkinson's and a variety of other neurodegenerative conditions and injuries. There is, therefore, a need for safe, scalable and reproducible technologies to expand populations of adult and embryonic stem cells to meet potential clinical need. Murine 3 T 3 feeder cells are commonly used for stem cell expansion. Although 'feeder-free' systems are being developed for a variety of stem cells, including embryonics, the use of feeder cells currently remains optimal for the expansion of epithelial stem cells.

The corneal epithelium is a rapidly regenerating stratified squamous epithelium. During homeostasis and following injury the corneal epithelium is regenerated from a distinct population of limbal epithelial stem cells (LESCs) believed to reside in the basal epithelium of the corneoscleral limbus [1-5].
LESC deficiency can occur as a result of chemical burns, Steven Johnson syndrome, or inherited eye disease such as aniridia. As a result of a partial or total depletion of the LESC pool, the adjacent conjunctival epithelium migrates over the surface of the cornea, causing pain, vascularisation and ultimately blindness [2-4].

In vitro laboratory expansion and transplantation of human LESC s can be a valuable aid in the treatment of LESC deficient patients. The most commonly used method for therapeutic in vitro LESC expansion involves the use of a growth-arrested murine fibroblast feeder layer and serum supplemented culture medium [6]. This method is based upon the original protocol by Rheinwald and $\mathrm{G}$ reen for the expansion of human epidermal keratinocytes [7]. The use of a feeder layer in the culture of limbal epithelial cells and the maintenance of their undifferentiated phenotype is highly dependant 
on a fibroblastic growth-arrested feeder layer, although a variety of alternative 'feeder-free' systems are being developed [8].

With growing concerns regarding the potential transmission of adventitious agents such as prions and animal viruses, it would be preferable to culture cells for human transplantation under xenobiotic-free conditions that can maintain stem cells. The aims of this study were to modify the culture of human limbal epithelial (HLE) cells in order to avoid the use of bovine serum and murine fibroblast feeder cells. HLE cells were cultured in the presence of growth arrested fibroblasts from different sources under serumsupplemented and serum-free conditions. M RC - 5 fibroblasts were selected for investigation owing to their precedence of use in the characterization and production of human viral vaccines [9-11].

\section{Materials \& methods}

Maintenance of 3T3 mouse fibroblasts and MRC-5 human embryonic fibroblasts 3T 3/J 2 mouse fibroblasts, a kind donation by Fiona Watts (C ancer Research UK), screened for mycoplasma since obtained, were maintained in Dulbecco's M odified Eagle M edium (DM EM ; Gibco) supplemented with $10 \%$ adult bovine serum (Gibco) and 1\% penicillin/streptomycin (Gibco).

M RC-5 human embryonic fibroblasts, originally established from normal lung tissue of a 14-week-old male fetus, were obtained from European Collection of Cell Cultures (ECACC) and were maintained in D M EM (G ibco) supplemented with $10 \%$ foetal bovine serum (FBS) (Gibco) and 1\% penicillin/streptomycin (Gibco). Culture medium was changed three times a week and the cultures were passaged upon reaching $60-70 \%$ confluence at a ratio of 1:8. The cultures were maintained at $37^{\circ} \mathrm{C}$ and $5 \% \mathrm{CO}_{2}$ in air. The fibroblasts were growth arrested in fibroblast culture medium containing mitomycin C (Sigma) for $2 \mathrm{~h}$ at a concentration of $4 \mu \mathrm{g} / \mathrm{ml}$ and $10 \mu \mathrm{g} / \mathrm{ml}$ for $3 \mathrm{~T} 3 / \mathrm{J} 2$ and M RC -5 fibroblasts, respectively.

Primary HLE cell isolation \& culture H LE cells were isolated from research-consented corneas supplied by M oorfields Eye Bank, London, UK. Primary cultures of HLE cells were isolated and maintained as described previously [12]. H LE cells were cultured in corneal epithelial culture medium (CECM ) consisting of DMEM F12 (1:1) (Invitrogen) supplemented with $10 \%$ FBS, $1 \%$ penicillin/streptomycin (Gibco), $0.1 \mathrm{nM}$ cholera toxin (Sigma), $5 \mu \mathrm{g} / \mathrm{ml}$ human recombinant insulin (Sigma), $0.05 \mathrm{mM}$ hydrocortisone (Sigma) and $10 \mathrm{ng} / \mathrm{ml}$ epidermal growth factor (Invitrogen). In the experiments described below, serum-free medium refers to CECM, as described above omitting the FBS. Culture medium was changed three times a week and the cultures were passaged upon reaching $70-80 \%$ confluence at a ratio of $1: 3$.

Cell morphology \& metabolic activity To determine the effect of serum-free culture upon the $3 T 3$ and MRC-5 feeder fibroblasts only, the cells were plated at a seeding density of $1 \times 10^{4} \mathrm{cells} / \mathrm{cm}^{2}$ in a 48-well plate. M etabolic activity was assessed using the Alamar Blue (AB) assay (Biosource) following the instructions by the manufacturer. To determine the metabolic activity of HLE cells co-cultured with growtharrested 3T 3 J2 mouse fibroblasts or M RC -5 human embryonic fibroblasts, $1.5 \times 10^{4}$ cell $\mathrm{s} / \mathrm{cm}^{2} \mathrm{HLEs}$ were seeded upon feeder layers prepared at a density of $2.4 \times 10^{4} \mathrm{cell} / \mathrm{s} / \mathrm{cm}^{2}$ and cultured in the presence and absence of serum. Before conducting the $A B$ assay the remaining fibroblast feeder cells were removed using $5 \%$ ethylenediaminetetraacetic acid (EDTA) solution (Sigma) for 2-5 min followed by gentle tapping. Subsequently, the $A B$ assay was carried out. Briefly, the culture media were removed and the wells were washed gently using PBS. The cells were incubated for $45 \mathrm{~min}$ at $37^{\circ} \mathrm{C}$ in $500 \mu \mathrm{l} A B$ reagent diluted $\times 10$ in $\mathrm{H}$ ank's balanced salt solution. After incubation, $150 \mu \mathrm{l}$ aliquots reagent from duplicate wells were transferred to a 96-well plate. Fluorescence was quantified at $560 \mathrm{~nm}$ excitation and $590 \mathrm{~nm}$ emission wavelengths (Zafire). The cell morphology was monitored using a Nikon inverted phase contrast microscope.

\section{Immunocytochemistry}

Eight-well Permanox chamber slides (Labtek, Nunk) were used for immunocytochemistry experiments. H LE cells were plated on growtharrested 3 T 3 or M RC -5 fibroblast feeder layers at seeding densities of $1.5 \times 10^{4} \mathrm{cells} / \mathrm{cm}^{2}$ and $2.4 \times 10^{4} \mathrm{cell} / \mathrm{s} / \mathrm{cm}^{2}$, respectively, and cultured in $400 \mu$ culture medium per well. $0 \mathrm{n}$ day 6 , the culture medium was removed; the cells were washed three times with PBS, fixed for $10 \mathrm{~min}$ at room temperature in $2 \%(\mathrm{wt} / \mathrm{vol})$ paraformaldehyde, and treated with 20\% (wt/vol) sucrose 
before storage at $-20^{\circ} \mathrm{C}$. The cultures were blocked for $1 \mathrm{~h}$ in PBS supplemented with $5 \%$ goat serum (Jackson) and $0.5 \%$ Triton $X$, followed by the primary antibody (mouse monoclonal antibody for $A B C G 2$ and mouse monoclonal antibody for cytokeratin 3, Chemicon International, and rabbit polyclonal antibody for $p 63 \alpha$, Cell Signalling, M A, USA) or blocking reagent only (negative control) overnight at $4^{\circ} \mathrm{C}$. Subsequently, the cells were incubated with their respective secondary antibody (goat antimouse fluorescein isothiocyanate (FITC), goat antimouse tetramethylrhodamine isothiocyanate (TRITC), goat antirabbit TRITC antibodies, respectively, all from Jackson), washed and counterstained with $2 \mu \mathrm{g} / \mathrm{ml}$ phalloidin FITC or TRITC accordingly (Sigma). Finally, the chamber slide wells were removed and the slides were mounted using Vectashield media with diamidino-phenylindole (DAPI) (Vector Labs). All incubations apart from the primary antibody incubation were performed at room temperature, and each step was interspersed with three 5-min rinses with PBS containing $0.1 \%$ tween- $20(\sigma)$.

\section{Colony-forming efficiency}

For the colony forming efficiency (CFE) assay, J2 fibroblasts were used as a feeder layer. The fibroblasts were treated with mitomycin $\mathrm{C}$ (M M C) as above and plated at a cell density of $4.8 \times 10^{5}$ cells in each well of a six-well plate and were allowed to attach for at least $2 \mathrm{~h}$. H LE cells were seeded at a density of 500 cells per well of the six-well plate. The medium was changed every other day. The cultures were observed closely from day 7 onwards and fixed when several colonies larger than $2 \mathrm{~mm}$ in diameter could be observed using a light microscope and graticule. Plates were fixed with cold methanol for $30 \mathrm{~min}$ at $-20^{\circ} \mathrm{C}$. Subsequently, the cells were rehydrated with PBS and stained with a solution of $1 \%$ rhodamine $B$ (Sigma) and $1 \%$ Toluidine Blue (Gurr) for $30 \mathrm{~min}$ at $37^{\circ} \mathrm{C}$. Finally, the plates were photographed using a light-box. Image J software was used to count the number of colonies that measured greater than $2 \mathrm{~mm}$ diameter. Colony-forming efficiency was calculated using the equation shown in Box $\mathbf{1}$

\section{Box 1. Calculation of the colony-forming efficiency.}

$$
\left(\text { Colony forming efficieny }(\%)=\frac{\text { Number of colonies }>2 \mathrm{~mm}}{\text { Number of cells seeded }}\right) \times 100
$$

Statistical evaluation

The statistical analysis of the results was carried out using Prism 3.0 (GraphPad, U SA). O ne-way analysis of variance (AN OVA) with Bonferroni post tests was used. All the experiments were performed in triplicate. All error bars represent standard error values.

\section{Results}

Fibroblast morphology \& proliferation

The proliferation of $\mathrm{J} 2$ and $M \mathrm{RC}-5$ fibroblasts was assessed using the $A B$ assay after 5 days in culture under serum-supplemented and serumfree conditions (Figre1). C ell metabolic activity indirectly indicating proliferation was unsurprisingly higher in the presence of serum compared with serum-free conditions for both fibroblast cell types. Fibroblast morphology and density was also monitored using light microscopy (Figre2). Under serum-free conditions the cultures appeared less confluent than those in the presence of serum. Moreover, cells cultured under serum-supplemented conditions exhibited a typical fibroblastic morphology whereas serum-free cultured cells showed an elongated stressed morphology. H owever, the effect of the lack of serum on the M RC-5 fibroblast morphology and proliferation was far less profound compared with the $3 \mathrm{~T} 3$ cells, indicating potential for the MRC-5 fibroblasts to survive and function under serum-free conditions (Figre2).

Feeder cell types affect HLE cell morphology \& proliferation

The levels of metabolic activity of H LE cells cultured on $\mathrm{J} 2$ and M RC - 5 fibroblast feeder layers were evaluated using the $A B$ assay after 5 days in culture under serum-supplemented $(\mathrm{J} 2+$ and MRC-5+) and serum-free (J2- and MRC-5-) conditions (Figre3). The assay was carried out after removing the feeder layers using EDTA. There were no significant differences in the levels of metabolic activity of the H LE cells in all different culture conditions and feeder layers, with the exception of M RC $-5+$ for which those levels were lower. Cell morphology was recorded using light microscopy on day 5 (Figure4).

As illustrated in Figure4, H LE cell morphology was influenced by the presence or absence of serum. Interestingly, in the presence of serum the M RC - 5 fibroblasts were unable to prevent H LE cell differentiation as indicated by the presence of large, flat, squamous-like cells (Figure4A), while in the absence of serum the HLE cells retained a small, undifferentiated morphology (Figure 4B). 
Figure 1. Metabolic activity of J2 fibroblasts (A) and MRC-5 fibroblasts (B) that have been cultured for 5 days in the presence $(+)$ or absence $(-)$ of serum.

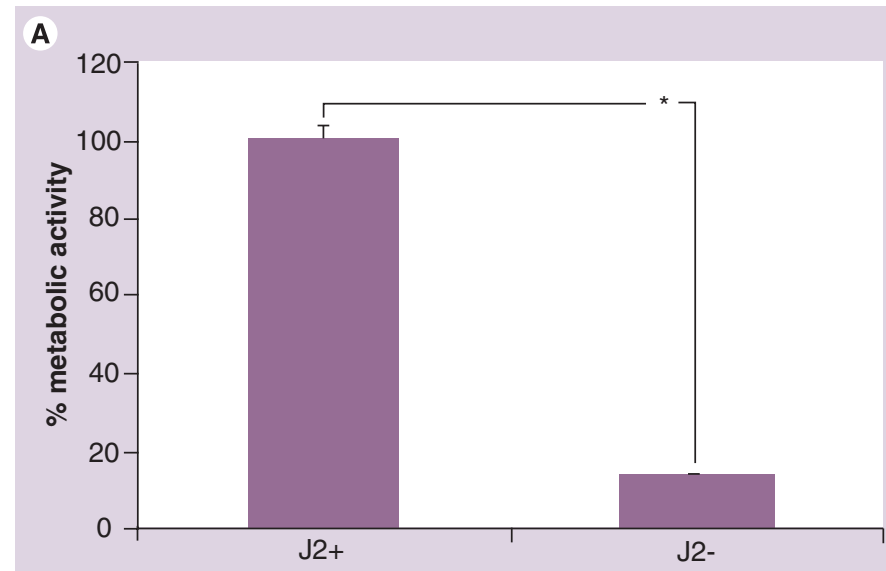

B

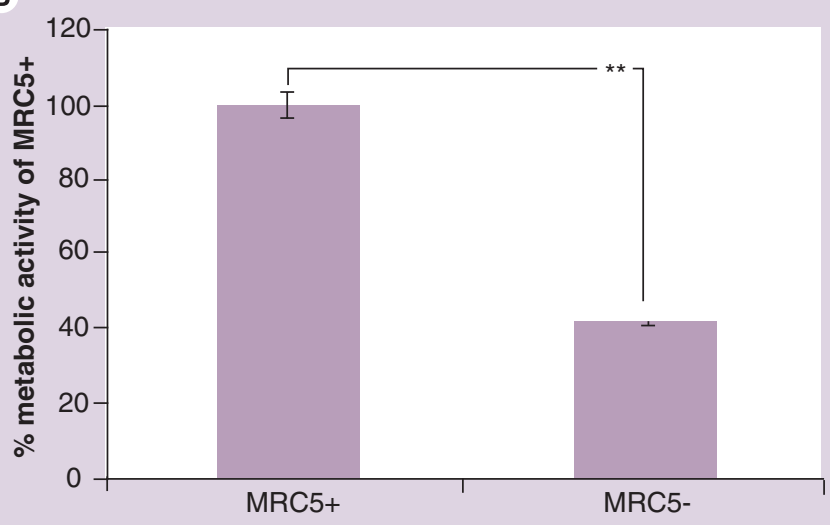

Error bars are standard errors of four replicates. on both M RC - 5 and $3 \mathrm{~T} 3$ feeder cells (Figure4B); however, expression levels were higher in serumfree cultures, especially for the M RC - 5 cultures. The squamous-like cells in all cultures tended to be negative or weakly stained for $P 63 \alpha$, indicating differentiation. The differentiation marker CK3 was predominantly present in M RC-5+ cultures, whereas only a low number of HLE cells in serum-free cultures were positive. In summary, expression levels of all markers as well as morphological characteristics were similar for HLE cells in both J2+and J2- and M RC - 5- culture types, a result correlating with the light microscopy observations.

\section{Colony-forming efficiency}

CFE assays were set up using secondary H LE cultures expanded on MRC-5 and $\mathrm{J} 2$ feeders in serum-supplemented and serum-free conditions. The HLE cells started forming colonies after day 5 postseeding. The experiments were terminated just before the colonies started touching, or up to 12 days from seeding. Results, illustrated in Figure6, showed that the HLE cells that were expanded in $\mathrm{J} 2+$ conditions had the highest clonal ability (CFE: $2.3 \%$ ) and M RC-5+ cultures had the lowest (CFE: $0.13 \%$ ). The M RC-5- cultures had a CFE of $1.46 \%$, a value close to the one of J2- cultures (CFE: $1.6 \%$ ), suggesting that although the assay was set up using H LE cells from secondary cultures, it was possible to retain and maintain stem cells on a M RC - 5 feeder layer under serum-free conditions.

This suggests that despite having low metabolic activity in serum-free medium (Figre1), once growth arrested, M RC -5 fibroblasts can successfully act as a feeder layer for H LE under serumfree conditions. The presence (Figure $3 C$ ) or absence of serum (Figre3D) did not appear to affect the ability of $3 \mathrm{~T} 3$ fibroblasts to maintain HLE cells of undifferentiated appearance.

\section{Immunocytochemistry}

Correlating with the morphology data, HLE cells co-cultured with M RC-5 feeder cells in the presence of serum were enlarged in size and did not express the putative LESC marker ABCG2, whereas those cultured with $M R C-5$ in the absence of serum were small, tightly packed and did express ABCG2 (Figre4A). This suggests that removal of serum from the M RC-5 coculture system is actually advantageous as it is less likely to induce HLE cell terminal differentiation. P63 $\alpha$ was expressed in H LE cells cultured

\section{Discussion}

The use of a feeder cell layer remains a requirement for the expansion of a variety of stem cells. In this work, the ability of MRC-5 human embryonic fibroblasts to sustain HLE cell culture was assessed and the potential to achieve this in serum-free conditions was demonstrated. A growing concern throughout European countries currently is that animal-derived adventitious agents such as bovine spongiform encephalitis (BSE) cannot be detected effectively using an in vitro diagnostic method. For that reason, a defined, serum-free alternative would be a preferred approach for therapeutic applications. This study is an encouraging advance towards xenobiotic-free cell culture.

It has been suggested that M RC -5 cells and fibroblasts can be grown in serum-free media (personal communication with Dr Glyn Stacey, N IBSC), but as yet serum-free media for fibroblast or keratinocyte culture with approval for 
Figure 2. Light microscopy images of MRC-5 and J2 fibroblasts that have been cultured for 5 days in the presence $(+)$ or absence (-) of serum.

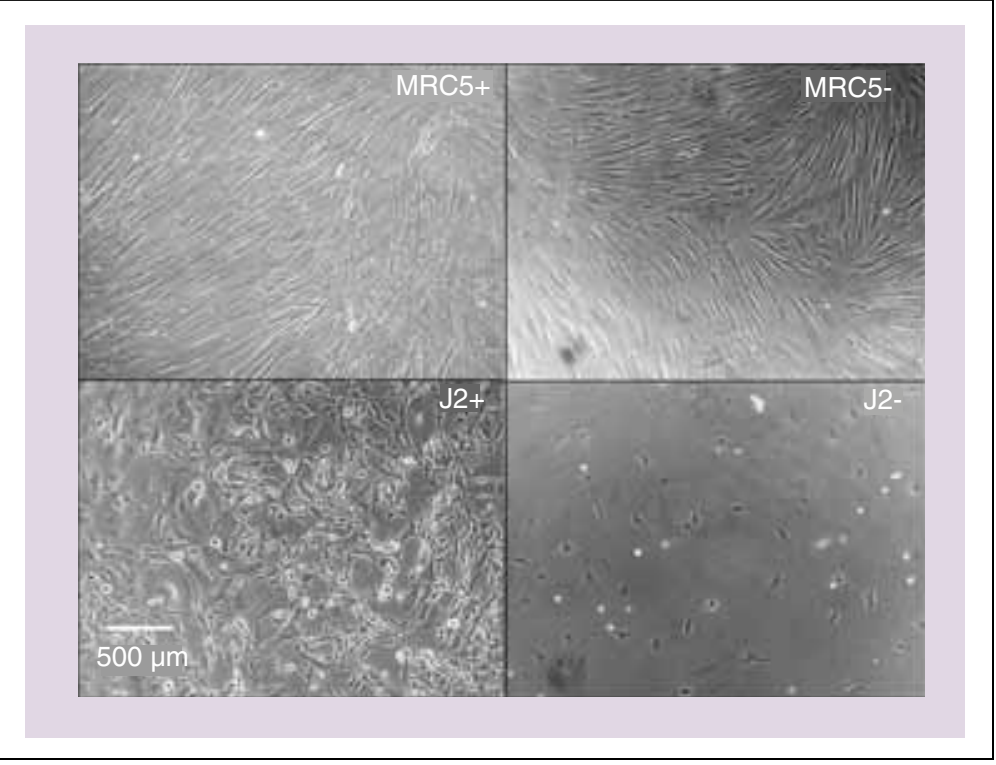

clinical use are not available. The regulatory route for serum-free media to be approved for clinical use is being explored by several media manufacturers. When this is available for fibroblast culture, the approach proposed in this paper for xenobiotic-free culture of HLE cells will be feasible; it is a reasonable expectation that this will occur within the next few years.

Figure 3. Metabolic activity of HLE cells cultured for 5 days on a feeder layer of either MRC- 5 or J2 ffbroblasts on $\mathbf{t} / \mathrm{c}$ plastic in the presence $(+)$ or the absence $(-)$ of serum.

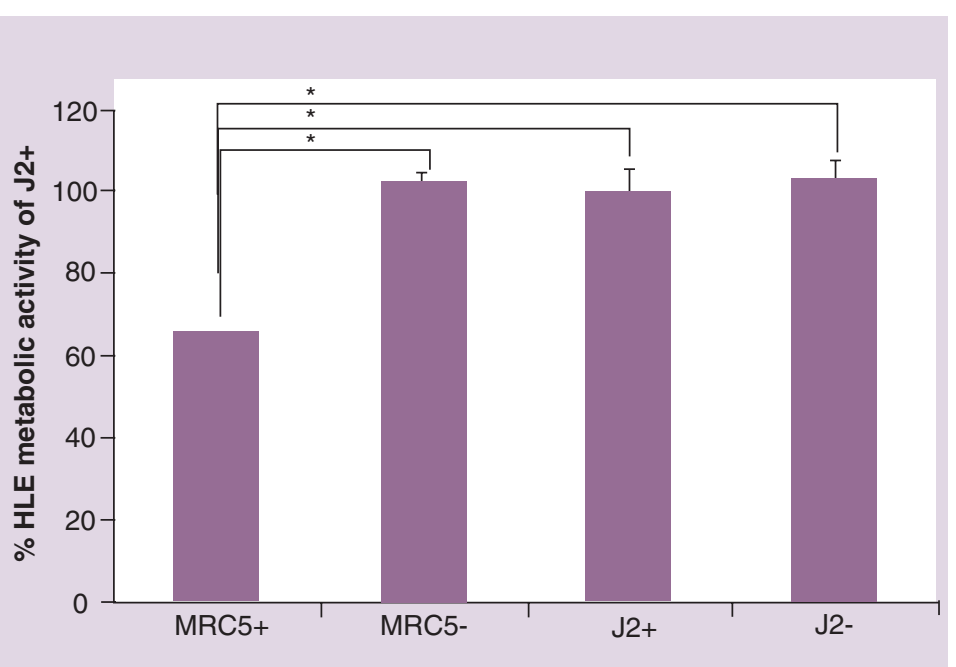

Error bars are standard errors of 4 replicates. HLE: Human limbal epithelial.
M RC - 5 fibroblasts have been shown to aid proliferation of various primary cell types when used as a feeder layer, for example, hepatocytes, since hepatocytes surrounded by mesenchymal cells in the mesoderma differentiate during embryogenesis [13]. H uman keratinocytes have also been cultured on M RC - 5 fibroblast feeder layers. Bosca et al. developed human skin equivalents in vitro with a unidimensionally retracted dermal equivalent made of human Type I and III collagen and human M RC-5 fibroblasts on which they cultured human keratinocytes and subsequently grafted those successfully in a mouse in vivo model [14]. Bullock et al. were the first to investigate a serum-free method for expansion of human keratinocytes using M RC -5 fibroblasts in a 3D plasma polymer scaffold [15]. The use of this human fibroblastic cell line as a feeder layer alternative for the culture of H LE cells has not until now been investigated.

The suitability of MRC-5 fibroblasts as a feeder layer for the culture and expansion of H LE cells was investigated by first assessing their metabolic activity levels, indicating proliferation on the different feeder layers in serum-supple mented versus serum-free conditions. The $A B$ assay data showed that HLE cells had significantly lower proliferation levels on MRC-5+ cultures than M RC-5-, J2+ and J2-. The other groups, apart from M RC $-5+$, did not show significant differences between their proliferation levels. This may be attributed to the early differentiation induced in the epithelial cells in the MRC-5+ cultures as determined by increased cell size and cytokeratin 3 expression. The reason for this is not yet understood, although it may be related to the observation that the M RC -5 cells seemed to adapt better to the absence of a lownutrient environment. M etabolic activity levels of M RC - 5 cells in the absence of serum were lower then serum-supplemented cultures but only by approximately a 2.5 -fold, as opposed to a fivefold decrease observed for 2 cells. The metabolic activity observations are also supported by the cell morphology data. The serum-free cultures of MRC-5 cells appear less stressed than the ones of $\mathrm{I} 2$ cells, which have lost their healthy characteristic fibroblastic appearance in the absence of serum.

Interestingly, it was demonstrated that HLE cells that were cultured on M RC - 5 cells largely differentiated in the presence of serum as opposed to serum-free conditions where low levels of HLE differentiation were observed as 


\section{Figure 4. Light microscopy images of HLE cells.}

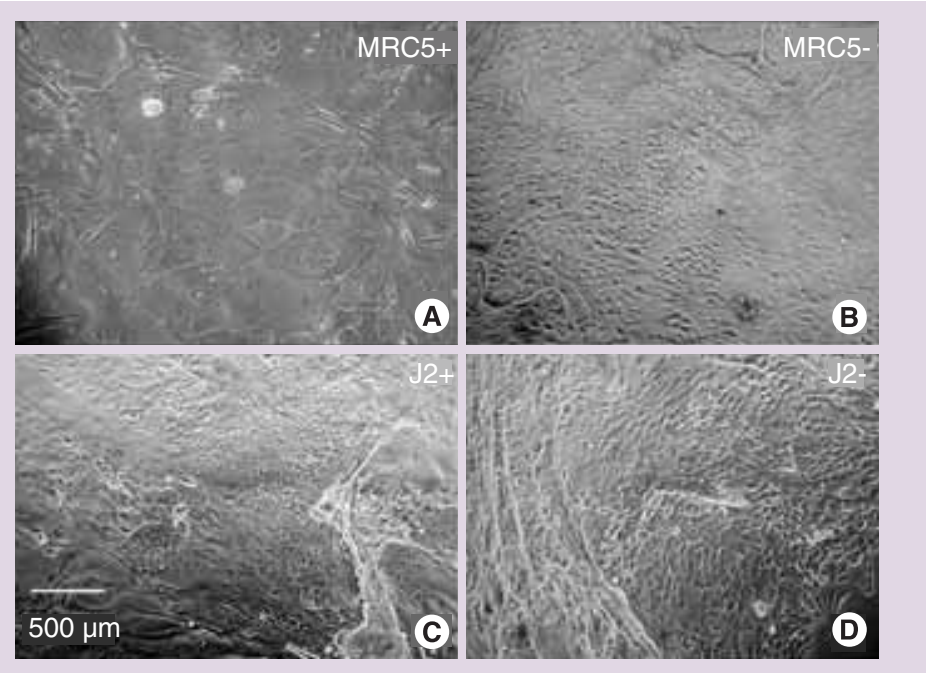

$(\mathbf{A}, \mathbf{B})$ Light microscopy images of HLE cells that have been cultured for 5 days on a feeder layer of MRC-5 fibroblasts, in the presence (+) or absence (-) of serum. (C,D) Light microscopy images of HLE cells that have been cultured for 5 days on a feeder layer of $\mathrm{J} 2$ fibroblasts, in the presence (+) or absence (-) of serum.

HLE: Human limbal epithelial. indicated by cell size and marker expression. Epithelial cells cultured on 12 cells exhibited a less differentiated phenotype in serum-free conditions but the difference with the serum-supplemented cultures was not as apparent. This suggests that the M RC -5 feeder cells in serumfree conditions may in fact be equivalent to $3 \mathrm{~T} 3$ feeders used in the presence of serum.

HLE cells in MRC-5+ cultures did not express ABCG2, whereas approximately $70 \%$ of cells in the other culture conditions including MRC-5- cultures were positive. Small, tightly packed epithelial cells were more brightly stained than enlarging, differentiating cells. The presence of a high percentage of brightly stained cells in the M RC-5-cultures is encouraging as studies on cultured primary HLE cells have correlated ABCG 2 expression and cell size with their clonogenic capacity [16]. The P63a transcription factor, a p53 homologue essential for regenerative proliferation in epithelial development and especially its $P 63 \alpha$ isoform, has also been used to distinguish human keratinocyte stem cells from their transit amplifying (TA) progeny [17,18]. M RC-5+ cultures of H LE cells showed partial expression of P63 $\alpha$ as opposed to the almost $100 \%$ positivity observed for the MRC-5-, J2+ and J2-cultures. Although this observation still supports the fact that the M RC-5+ cultures exhibited the most differentiated phenotype, it also agrees with other studies showing that p63 $\alpha$ is expressed in a large percentage of H LE cells in culture as opposed to its in vivo expression, which suggests that it is a less preferred option as a H LE stem cell marker [19], unless the cell size and the nucleus: cytoplasm ratio is also taken into account [20]. Furthermore, in a number of studies $A B C G 2$ has been shown to indicate the location of LESC s in the basal epithelial layers of the limbus where clusters of positive cells are observed [21-23]. H owever, in this study many more HLE cells expressed $A B G G 2$ than would be expected if this was a true LESC marker in vitro. This suggests that in vitro expression of 'stem cell markers' on cultured cells alone should be interpreted only as an indicator of the presence of early transient amplifying cells. Further characterization would still be required to identify LESC s. C orrelating with these findings, $100 \%$ of $\mathrm{HLE}$ cells in the MRC-5+ cultures expressed the differentiation marker cytokeratin 3 , whereas very few cells in all other conditions were cytokeratin 3 positive.

$N$ evertheless, the high level of expression of the ABCG 2 and P63 $\alpha$ shows promise that the HLE cells retain a less differentiated phenotype correlating with the in vivo phenotype in basal LESC s in vivo [22]. H owever, it does not provide evidence that all of the positive cells are stem cells. The CFE assay is used to enhance and supplement the results from the putative stem cell markers $A B C G 2$ and $P 63 \alpha$, as a more specific stem cell marker remains elusive. In this work, the CFE assay was used according to Pellegrini et al. [24]. The assay results in this study demonstrated the ability of M RC -5 fibroblasts to sustain H LE stem cells that formed colonies larger than $2 \mathrm{~mm}$ at a rate of $1.8 \%$, suggesting that they were holoclone colonies. Although it must be noted that the $\mathrm{J} 2$ fibroblasts supported higher clonal ability of the HLE cells (CFE: 2.3\%), the value for the M RC-5- conditions of culture is still satisfactory, especially given the fact that the HLE cells used for the assay originated from secondary cultures. It is worth noting that these secondary cultures were carried out in a T 25 flask using the feeder density as recommended for 3 T 3 cells $\left(2.4 \times 10^{4}\right.$ cells $\mathrm{cm}^{-1}$ ), indicating that the M RC-5 fibroblasts can successfully sustain H LE cell cultures on a larger scale than would be required clinically under serum-free conditions. 
Figure 5. Immunocytochemistry of HLE cells cultured for 5 days on a feeder layer of either MRC-5 or $\mathbf{2}$ fibroblasts in the presence ( + ) or absence (-) of serum.

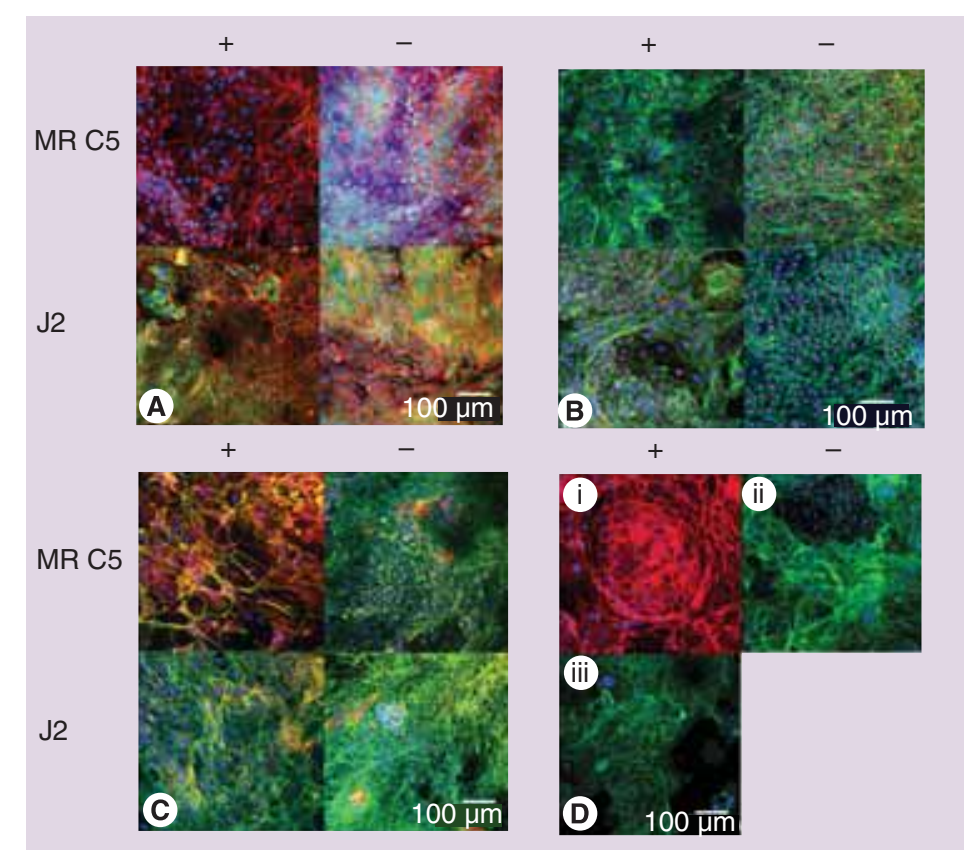

(A) ABCG2- green, phalloidin FITC-red, DAPI-blue (B). $\triangle \mathrm{NP63} \alpha$-red, phalloidin FITC-green, DAPI-blue (C) Cytokeratin 3-red, phalloidin FITC-green, DAPI-blue DAPI: Diamidino-phenylindole; FITC: Fluorescein isothiocyanate; HLE: Human limbal epithelial.
All of the data discussed suggests that the presence of serum in the M RC -5 coculture system induces high levels of differentiation of HLE cells. 0 ther workers have shown that serum can
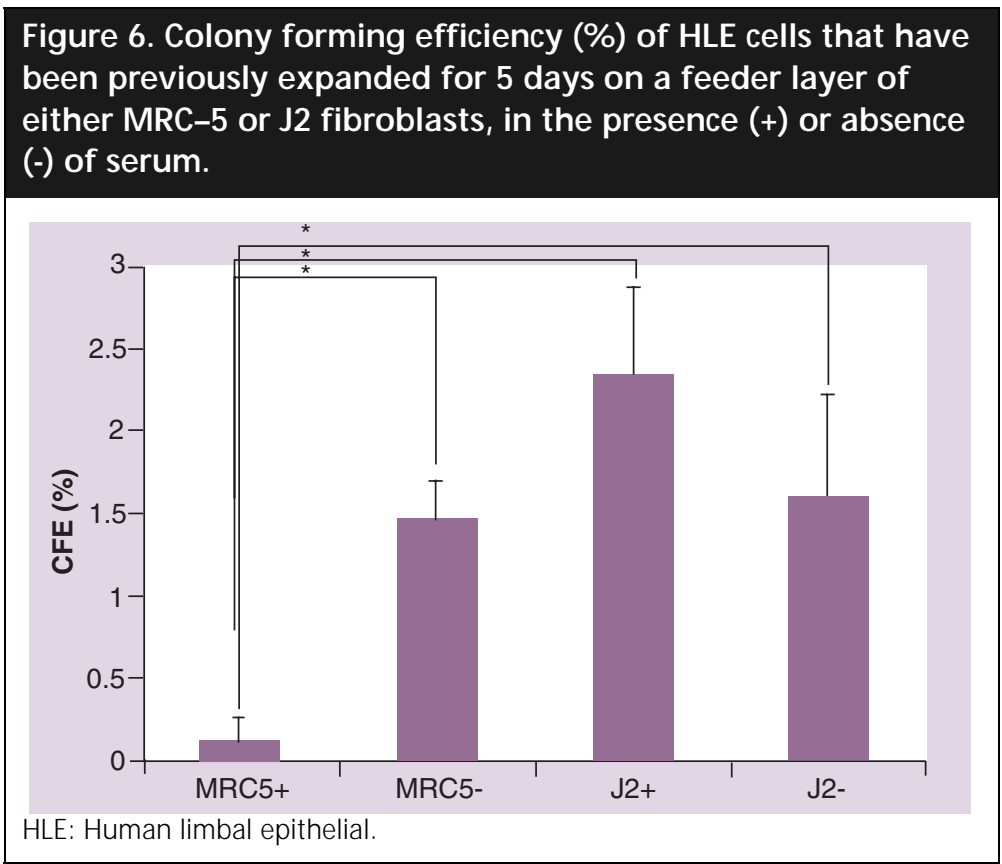

affect limbal epithelial cell proliferative capacity and differentiation: Kurse and Tseng demonstrated that increasing percentages of serum in the culture medium can increase the C FC of rabbit limbal epithelial cells, in contrast to corneal epithelial cells, in which the opposite effect was observed, suggesting serum contains factors that stimulate LESC into clonal proliferation $[25,26]$. $\mathrm{H}$ owever, the fact that the largely differentiated M RC-5+cultures exhibit significantly lower levels of CFE and proliferation than the $\mathrm{J} 2+, \mathrm{J} 2$ and MRC-5- cultures demonstrates that the MRC-5 cell line does not correlate with these reports. The reason behind these differences may lie in the cytokine expression profile of the M RC - 5 cell line when stimulated by serum and is currently under investigation. H owever, given the controversy over the use of serum, it can be safely suggested that the exclusion of animal serum is a definite advantage for clinical applications, especially because as far as we are aware this is the first study to demonstrate the ability of a human fibroblast feeder cell line to sustain H LE cell cultures in the absence of serum. The presented data indicate important differences between M RC -5 and $J 2$ cells and show that the MRC-5 cell can maintain stem cells under serum-free conditions. Encouragingly, within 5 days of serum-free co-culture with the M RC -5 cells, sufficient numbers of H LE's could be generated for application onto a human cornea. H ence the M RC-5 cell line is worth exploring for the xenobiotic-free expansion of LESC $s$ and other stem cells.

\section{Conclusions}

MRC -5 human embryonic fibroblasts were shown to be suitable for use as feeders for H LE cell culture in serum-free conditions. The H LE cells used under those conditions expressed high levels of putative stem cell markers $A B C G 2$ and P63 $\alpha$ and low levels of the differentiation marker CK3. Moreover, CFE data showed that M RC -5 cells can form holoclones when cultured in serum-free conditions, indicating that they can sustain the stem cell phenotype. These data show promise that this cell line could potentially be used clinically for corneal regeneration applications.

\section{Financial \& competing interests disclosure}

The authors gratefully acknowledge the UK Government's Biotechnology and Biological Sciences Research Council (BBSRC) for their support and funding under the Small Business Research Initiative (SBRI) scheme and also the 
Special Trustees of M oorfields Eye Hospital. The authors have no other relevant affiliations or financial involvement with any organization or entity with a financial interest in or financial conflict with the subject matter or materials discussed in the manuscript apart from those disclosed.

$\mathrm{N}$ o writing assistance was utilized in the production of this manuscript.

\section{Ethical conduct of research}

The author states that they have obtained appropriate institutional review board approval or have followed the princ ples outlined in the Declaration of Helsinki for all human or animal experimental invesigations In addition, for investigations involving human subjects, informed consent has been obtained from the participants involved.

\section{Executive summary}

M RC-5 cells, a human embryonic cell line, can sustain limbal epithelial cell expansion under serum-free conditions

When serum is present this cell line drives limbal epithelial cell differentiation

Metabolic activity assessment showed that human limbal epithelial cells cultured serum-free on a M RC-5 fibroblast feeder layer proliferated as well as cells cultured serum-free on J2 cells.

MRC -5 fibroblasts under serum-free conditions expressed high levels of the putative stem cell markers ABCG2 and P63 $\alpha$ and low levels of the differentiation marker CK3, indicating that they retained a 'stem cell-like' phenotype under those culture conditions.

Cells expanded on both M RC - 5 and $\mathbf{2} 2$ fibroblasts in serum-free conditions had a colony-forming efficiency of approximately $1.5 \%$, indicating the maintenance of stem cells.

These results demonstrate plausibility of expanding human limbal epithelial cells for clinical applications by using a human fibroblast cell line as a feeder layer, excluding the use of bovine serum, while preserving the proliferative potential and stem cell characteristics of human limbal epithelial cells.

\section{Bibliography}

1. Stepp M A, ZieskeJD : T he corneal epithelial stem cell niche. 0 cul. Surf. 3(1), 15-26 (2005).

2. Dua HS, Shanmuganathan VA, PowellRichards AO, Tighe PJ, Joseph A: Limbal epithelial crypts: a novel anatomical structure and a putative limbal stem cell niche. Br. J. O phthalmol. 89(5), 529-532 (2005).

3. D aniels JT, D art JKG, Tuft SJ, Khaw PT: Corneal stem cells in review. Wound Repair Regen. 9(6), 483-494 (2001).

4. Tseng SC G, Li D Q : D ifferential roles of keratinocyte growth factor and hepatocyte growth factor scatter factor in regulating corneal epithelial stem cells and transient amplifying cells. Mol. Biol. Cell 7, 3087-3087 (1996).

5. Cotsarelis G, Cheng SZ, D ong G, Sun TT, Lavker RM : Existence of slow-cycling limbal epithelial basal cells that can be preferentially stimulated to proliferate implications on epithelial stem-cells. Cell 57(2), 201-209 (1989).

6. Pellegrini $G$, Traverso CE, Franzi AT et al.: Long-term restoration of damaged corneal surfaces with autologous cultivated corneal epithelium. Lancet 349(9057), 990-993 (1997).

7. Rheinwald JG, Green $\mathrm{H}$ : serial cultivation of strains of human epidermal keratinocytes formation of keratinizing colonies from single cells. Cell 6(3), 331-344 (1975).
8. Lindberg K, Brown M E, Chaves H V, Kenyon $K R$, Rheinwald JG : In vitro propagation of human ocular surface epithelial-cells for transplantation. Invest. O phthalmol. Vis. Sci. 34(9), 2672-2679 (1993).

9. Jacobs JP, Jones CM, Baille JP: Characteristics of a human diploid cell designated M RC - 5. N ature 227(5254), 168-170 (1970).

10. Jacobs JP: Status of human diploid cell strain M RC -5 as an approved substrate for production of viral vaccines. J. Biol. Stand. 4(2), 97-99 (1976).

11. Rosolowsky M, M cKee R, Nichols W, Garfinkle B: Chromosomal characterization of M RC - 5 cell banks utilizing G -banding technique, In: Safety of Biological Products Prepared from M ammalian Cell Culture. 109-117 (1998).

12. N otara M, Bullett N A, D eshpande P et al.: Plasma polymer coated surfaces for serumfree culture of limbal epithelium for ocular surface disease. J. M ater. Sci. M ater. M ed. 18(2), 329-338 (2007).

13. Dodd I, Browne M J, Robinson JH : Identification of the calcium-inducible plasminogen activators secreted by a humandiploid fibroblast cell-line (M RC-5). Thromb. Res 40(5), 693-702 (1985).

14. Bosca $A R$, Tinois $E$, Faure $M$ et al.: Epithelial differentiation of human-skin equival ents after grafting onto nude-mice. J. Invest. D ermatol. 91(2), 136-141 (1988).
15. Bullock AJ, H igham M C, M acneil S: U se of human fibroblasts in the development of a xenobiotic-free culture and delivery system for human keratinocytes. Tissue Eng. 12(2), 245-255 (2006).

16. De Paiva CS, Pflugfelder SC, Li D Q : C ell size correlates with phenotype and proliferative capacity in human corneal epithelial cells. Stem Cells 24(2), 368-375 (2006).

17. Di lorio E, Barbaro V, Ruzza A et al.: I soforms of D elta N p63 and the migration of ocular limbal cells in human corneal regeneration. Proc. N atl Acad. Sci. U SA 102(27), 9523-9528 (2005).

18. Pellegrini G, D ellambra E, Golisano 0 et al.: p63 identifies keratinocyte stem cells. Proc. N atl Acad. Sci. U SA 98(6), 3156-3161 (2001).

19. Salehi-H ad H, Alvarenga LS, Isseroff $R$, Schwab IR: Factors modulating p63 expression in cultured limbal epithelial cells. Cornea. 24(7), 845-852 (2005).

20. Arpitha P, Prajna N V, Srinivasan M , M uthukkaruppan $\mathrm{V}$ : H igh expression of p63 combined with a large $N / C$ ratio defines a subset of human limbal epithelial cells: Implications on epithelial stem cells. Invest. 0 phthalmol. Vis. Sci. 46(10), 3631-3636 (2005).

21. De Paiva CS, Chen Z, Corrales RM, Pflugfelder SC, Li D Q : ABC G2 transporter identifies a population of clonogenic human limbal epithelial cells. Stem Cells23(1), 63-73 (2005). 
A xenobiotic-free culture system for human limbal epithelial stem cells - RESEARCH ARTICLE

22. Watanabe $\mathrm{K}, \mathrm{N}$ ishida $\mathrm{K}$, Yamato $\mathrm{M}$ et al.: $H$ uman limbal epithelium contains side population cells expressing the AT P-binding cassette transporter $A B C G 2$. FEBS Letters 565(1-3), 6-10 (2004).

23. Budak MT, Alpdogan OS, Zhou MY et al.: 0 cular surface epithelia contain ABC G2dependent side population cells exhibiting features associated with stem cells. J. Cell. Sci. 118(8), 1715-1724 (2005).
24. Pellegrini G, Golisano O, Paterna P et al.: Location and clonal analysis of stem cells and their differentiated progeny in the human ocular surface. J. Cell Biol. 145(4), 769-782 (1999).

25. Kruse FE, Tseng SC G : Serum differentially modulates the clonal growth and differentiation of cultured limbal and corneal epithelium. Invest. 0 phthalmol. Vis. Sci. 34(10), 2976-2989 (1993).
26. Kruse FE, Tseng SCG : A serum-free clonal growth assay for limbal, peripheral, and central corneal epithelium. Invest. O phthalmol. Vis. Sci. 32(7), 2086-2095 (1991). 